\title{
Granular flow in equilibrium with the bottom: experimental analysis and theoretical prediction
}

\author{
B. Zanuttigh and A. Lamberti \\ DISTART - Universitá di Bologna Viale del Risorgimento 2, 40136 BOLOGNA, Italy
}

Received: 10 November 2000 - Revised: 15 June 2001 - Accepted: 2 August 2001

\begin{abstract}
The paper presents measurements performed on the granular flow that develops in a drum partially filled with sand grains and rotating at various speeds. The aims of the paper are: to provide experimental evidence and measurements on grain flow in a drum; to compare theoretical and experimental velocity profiles; to point out discrepancies among theory and experiments.

Velocity and "temperature" profiles were obtained with a Laser Doppler Anemometer (LDA) in the mid-section of the stream, where the flow is usually uniform; image analysis and visual observations of the flow were also carried out to evaluate the local slope, the depths of the characteristic flow regions and the concentration of the granular material. A semi-empirical relation that fits the experimental velocity profiles is presented and compared with Takahashi's velocity distributions for rigid and erodible bed.

As proven by the distributions of free surface elevation, velocity, volumetric concentration and grain size across the drum, the three-dimensional nature of the flow field is not negligible.

By increasing the drum rotation speed, in correspondence with critical and supercritical flows, changes in the flow regime are observed with formation of quasi-stationary surface waves. Wave development is described by analysing the extension and form of the experimental and theoretical velocity profiles. Wave effects on measurements are quantified and checked comparing the free-surface velocity-discharge relation obtained from experiments and from Takahashi's model for erodible bed.
\end{abstract}

\section{Introduction}

Granular flows over a homogeneous erodible bottom are of particular interest because they represent the shearing conditions in which natural debris flows often develop.

Correspondence to: B. Zanuttigh

(barbara.zanuttigh@mail.ing.unibo.it)
The experimental analysis of the flow of dry granular materials and granular mixtures is appropriately synthesised in the monograph of Takahashi (1991), who analysed the velocity field that develops both over a rigid and over an erodible bottom. Different approximations of velocity profile were determined in the two cases and it was shown that for a granular flow in equilibrium with the immobile bed phase, variations of grain concentration and friction angle along the vertical cannot be neglected. Granular flow over an erodible bottom have been recently examined by Egashira et al. (2000) who developed a constitutive model that highlights the differences in velocity and volumetric concentration profiles of a mixture over a rigid and an erodible bottom.

Whilst Egashira evaluates the equilibrium condition as the upper concentration limit that allows for all the material to flow over a rigid bottom, in this study we impose in the facts that the flowing material is in equilibrium with the bottom and we analyse the velocity field that develops in a drum partially filled with dry sand grains, rotating at different speeds (see Zanuttigh, 1998).

Partially filled rotating drums have many practical applications in material processing, as surface polishing and grain drying. Moreover, they are easy to build and control and are able to generate a continuous stationary grain flow in equilibrium over a stationary bottom, characterised by all the possible regimes of motion. First, Franklin and Johanson (1955) used this device to measure the angle of repose of granular materials and observed different free-surface shapes depending on the cohesion of the tested material. Later, Cantelaube and Bideau (1995) analysed, following the trajectories of tracing particles, the radial segregation process in a drum partially filled with smooth disks of two different diameters. Boateng and Barr (1996) performed measurements of velocity, concentration and "temperature" using fiber-optic sensors in a drum filled with a large variety of materials, as polythene, rice and limestone.

The objectives of the present study are to determine a relationship between discharge, depth and slope of the flow and to characterise experimentally the flow as precisely as possi- 


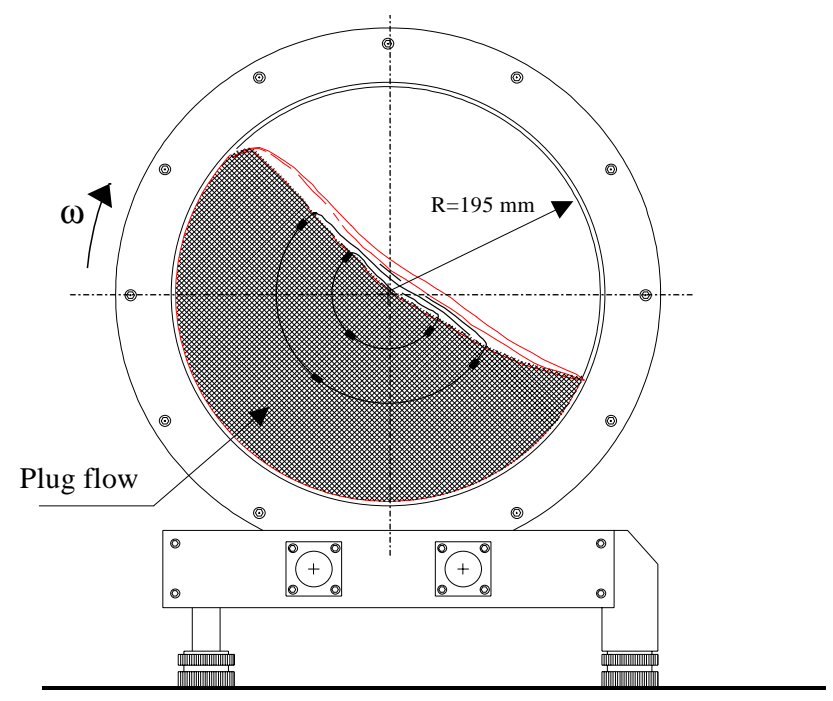

Fig. 1. Front view of the rotating drum.

ble, in order to check the agreement with flow fields derived from constitutive granular models.

The paper is composed of three main parts. The first part describes the experimental setup and with some preliminary tests demonstrates the validity of the LDA as the adopted acquisition technique.

The second part presents the principal experimental results: velocity and "temperature" (granular temperature is the trace of velocity covariance tensor) profiles, which were obtained with LDA measurements close to the wall, at a limited distance from the wall and on the surface across the drum. Visual observations and image analysis allowed one to evaluate the slope and the volumetric concentration of the flow. The presence of different flow regions is shown and criteria to distinguish the characteristic flow depths are determined. Three-dimensional effects of the geometry on the flow are observed, showing that the dimensions of the device with respect to the flow stream play an important role on measurements, as pointed out by several authors (but usually disregarded: see, for instance, Lanzoni and Tubino, 1993).

The third part relates experimental results to theoretical approximations. A semi-empirical relation that fits well with the experimental velocity profiles is presented and is compared with velocity profiles that follow Takahashi's laws. Effects of free-surface waves on velocity profiles are deeply analysed. The experimental relationship between the discharge, depth and slope of the flow is compared with the relation obtained by Takahashi for dry granular flows, and discrepancies between experimental results and theoretical predictions are widely discussed.

\section{Experimental setup and measurements technique}

The experimental equipment consists of a cylinder with inner diameter of $390 \mathrm{~mm}$ and axial length of $131 \mathrm{~mm}$ (front view

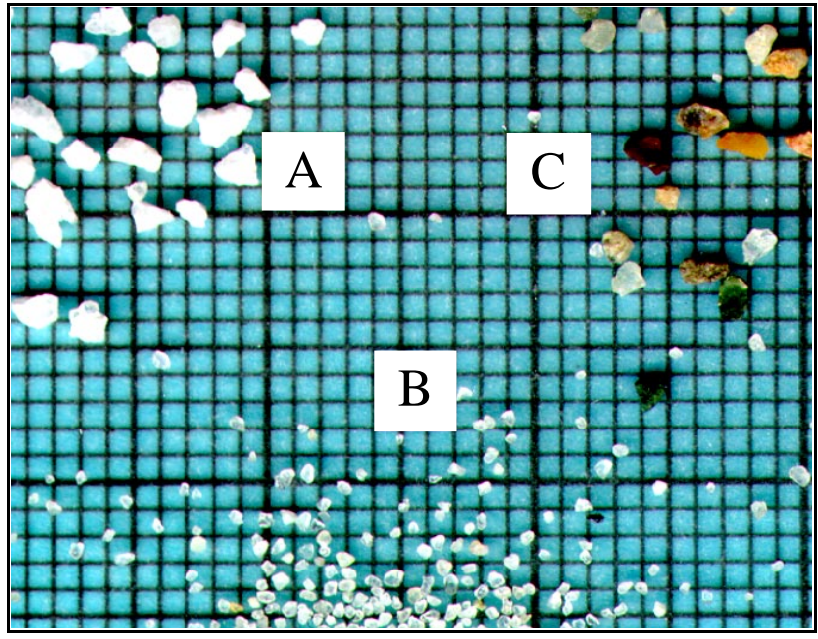

Fig. 2. Irregular shape tested materials.

in Fig. 1), which is mounted on a pair of friction rollers and rotates around its axis at a constant speed in the range 0$10 \mathrm{rpm}$. The drum front and back walls are made of $10 \mathrm{~mm}$ thick glass to allow for optical measurements; the internal surface of the cylinder was made rough with sandpaper to avoid the tendency of the whole bed to slide on the boundary as a solid body.

The drum can be filled with dry grains, grains and water (or any fluid), in order to analyse grain-fluid mixture behaviour. Many tests with glass spheres and natural sand of irregular shape (some of the materials are shown in Fig. 2) were performed; in Table 1, a summary of the material characteristics and test conditions is reported. For smaller particles, instability can arise and approximately stationary conditions cannot be obtained.

In the tests described here, the drum is filled with natural sand grains of $0.84-1.19 \mathrm{~mm}$ diameter, material $C$ in Fig. 2; similar tests were performed with the other materials reported in Table 1: results are not discussed in the present paper but confirm the qualitative conclusions drawn. The experiments were carried out at different rotation speeds in the range 1-6rpm. Three kinds of measurements were performed in correspondence with the mid-section of the stream, where the flow is usually uniform:

- visual observations to evaluate the local slope and the characteristic depths of the flow;

- image analysis at the wall and on the surface across the axial length of the stream to evaluate the concentration in volume and the local grain size;

- LDA measurements to obtain velocity and grain "temperature" profiles close to the wall (at a distance of $1.5 \mathrm{~mm}$ from the wall), at the maximum distance $(5.5 \mathrm{~mm})$ from the wall that allows for reliable measurements, and on the free surface. 
Table 1. Tested materials and test conditions. Sand grains B' have been obtained by sieving the materials B. $\rho_{s}$ is the material density, $\Phi_{r}$ is the maximum slope angle before avalanching, $\Phi_{s}$ is the slope angle after avalanching, $C_{0}$ is the maximum grain volume concentration obtained under vibration, $\omega$ is the drum rotation speed

\begin{tabular}{lccccc}
\hline Material & $\rho_{S}(\mathrm{~kg} \mathrm{~m}-3)$ & $\Phi_{r}\left({ }^{\circ}\right)$ & $\Phi_{s}\left({ }^{\circ}\right)$ & $C_{0}$ & $\omega(\mathrm{rpm})$ \\
\hline Sand grains 1.2-1.7 mm (A) & 2520 & $35.1 \pm 0.2$ & $30.9 \pm 0.6$ & 0.58 & 5 \\
Sand grains 0.1 mm & - & - & - & - & unstable \\
Sand grains 0.2-0.4 mm (B) & - & - & - & - & unstable \\
Sand grains 0.3-0.4 mm (B') & - & - & - & - & unstable \\
Sand grains 0.84-1.19 mm (C) & 2530 & $33.5 \pm 0.2$ & $30.1 \pm 0.4$ & 0.57 & $2-5$ \\
Glass beads 0.2-0.3 mm & - & - & - & - & unstable \\
Glass beads 0.3-0.4 mm & 2410 & $23.3 p m 0.2$ & $22.5 \pm 0.2$ & 0.62 & $2-5$ \\
\hline
\end{tabular}

Visual observations were performed using transparent sheets, on which free-surface envelopes and the flow lower boundary (see Sect. 3.1) were drawn together with the centre of the drum and the horizontal, which were taken as a reference system for the following evaluations.

The image analysis was carried out on digital photos taken at different speeds, along the vertical and across the channel (the channel is the space between the drum walls where grain flow takes place); the concentration in volume was estimated by counting the number of particles per unit area.

Measurements with LDA were performed with two different LDA displacements. Measurements along the vertical were performed by vertically moving the LDA, kept at a given distance from the drum's front wall and horizontally fixed.

For measurements on the free-surface, across the channel, the LDA was placed at an elevation higher than the flow freesurface and was inclined at $45^{\circ}$ with respect to the horizontal, in order to measure from above. The instruments were moved towards the drum to reach the desired distance from the wall; then, at a given distance, the measurement points were obtained moving the LDA along the vertical.

The adopted LDA is characterized by a measure volume of $80 \mu \mathrm{m} \times 680 \mu \mathrm{m}$, a fringe spacing equal to $4.2 \mu \mathrm{m}$ and a focal length of $160 \mathrm{~mm}$. LDA is quite unusual for measuring the velocity of particles whose size is of the order of the measure volume.

The validity of the output signal from the LDA and the reliability of the measuring system was, therefore, preliminary tested. The test setup consisted of a cylinder and a sheet, on which grains were glued with a known concentration. This sheet was fixed, all along the circumference, on the cylinder, rotating at constant speed around its vertical axis. Particle velocities were measured with the LDA at different rotation speeds, changing the voltage and the bandwidth of the photomultiplier. Tests were performed using all three bandwidth available $( \pm 0.16 \mathrm{~m} / \mathrm{s}, \pm 0.54 \mathrm{~m} / \mathrm{s}, \pm 1.62 \mathrm{~m} / \mathrm{s})$ and different voltages in the range $600-900 \mathrm{~V}$.

The analysis of the results showed some fundamental criteria on the choice of the bandwidth and the voltage. The best voltage must not be too low, in order to have a high data rate and a high validation ratio (validated data not less than the $90 \%$ of the acquired data), and not too high, in order to avoid saturation. The measured velocities appear slightly dependent on modest variation of the voltage in the range of the best one; for instance, in the case of the tested material, the best output was obtained with a voltage equal to $700 \mathrm{~V}$, which has been chosen after some preliminary measurements performed at $600 \mathrm{~V}$ and $800 \mathrm{~V}$. The dependence of the measurements on the bandwidth is more important: in order to have the correct output, the bandwidth must be chosen so that the velocity values to be acquired are all inside it. Thus, the bandwidth must have approximately an amplitude equal to twice the amplitude of the maximum velocity to be measured: in this case, the values of the velocity variance are minimum. Considering the results obtained with the correct bandwidth and the best voltage, the agreement between the theoretical and the mean measured values was always within $1 \%$; the standard deviation of measurements is about $4 \%$ instead of zero, but it should be noted that the rotation of the device was not controlled by a feed-back system, and small oscillations of the rotation speed with relative amplitude of $\sim 2 \%$ and a positive drift of $\sim 1 \%$ (after 3 hours) were measured. In conclusion, the measuring technique is reliable and introduces some noise in the measurements, which under the best experimental conditions are around $2 \%$ of the mean observed values.

Apart from our validation tests, Schippa and Lamberti (1999), using the same LDA performed tests on a rotating annular rheometer, obtained velocity measurements consistent with the adherence at the upper and lower rigid surfaces. Durst (1987) and Naqwi and Durst (1991, 1992) showed that the Doppler technique may be used for the sizing of small particles as well as large particles in any case. Taniere et al. (1997) performed measurements of heavy solid particles in a turbulent air boundary layer, obtaining velocity values in agreement with a selected 2D model. Experimental observations and a general theory on precision sizing of large moving particles using diffraction splitting of Doppler lines were carried out by Kononenko et al. (1999). The équipe of Rasmussen (1996), University of Aarhus, performed LDA velocity measurements in wind tunnels on different large sand particles in order to study wind erosion, sediment transport and dune dynamics. 
Concerning the distance from the wall at which LDA measurements are reliable, with the same LDA, Schippa and Lamberti (1999) could see up to 3 grain layers; in the drum, the concentration close to the wall is lower and penetration up to 5 grains was possible. Measurements along the vertical were performed at several distances from the wall, between $1.5 \mathrm{~mm}$ and $5.5 \mathrm{~mm}$. A regular trend in these measurements was found: velocities and flow depths increase with increasing distance, which is consistent with wall adherence and internal friction. In these measurements, we doubt the possible influence of energy scattered by particles in the region between the wall and beam intersection. In this sense, the quality of measurements decreases with increasing distance from the wall: the measurement point might not exactly correspond to beam intersection (or focus of the optical system) but to a point at a lower distance from the wall; if this is the case, side wall effects at a greater distance from the wall are actually underestimated. Obviously, the LDA data rate is rapidly decreasing with decreasing distance of the measure volume from the front wall; at $5.5 \mathrm{~mm}$ the data rate was very low, but the validation fraction of the acquired data was very high, from $95 \%$ to $100 \%$. In the present paper, only a set of measurements is reported, which corresponds to the maximum distance from the wall that guaranteed high validation and a reasonable data rate.

\section{Qualitative results}

This section presents the experimental results obtained by visual observations, image analysis and LDA measurements. Section 3.1 gives a qualitative description of the flow development and defines criteria to distinguish the different flow regions. Then, in Sect. 3.2, the flow characteristics are deeply analysed through velocity and concentration profiles and grain-size distribution across the channel. Results show relevant side wall effects. Finally, the attention in Sect. 3.3 is focused on the change in flow-regime that occurs by increasing the drum speed, with formation of stationary free-surface oscillations.

\subsection{Description of the flow}

The flow regime is characterised by different regions, which are reported in Fig. 3. In the lower part of the drum a plug flow develops with packed grains moving along circular paths around the rotation axis. The active layer is a shearing region that lies over the plug flow; it can be divided into a quasi-static lower layer, characterised by a slow flow and multiple contacts between the particles, and a dynamic upper layer, with grain motion in a kinetic collisional regime. Close to the free-surface, stationary oscillations of the free-surface profile, more evident with increasing rotation speed, cause an intermittent presence of material at the measure point.

The flow slope results are nearly constant, varying in a range of $3^{\circ}$ from low to high drum speeds. Characteristic flow depths, which were evaluated following the criteria ex-

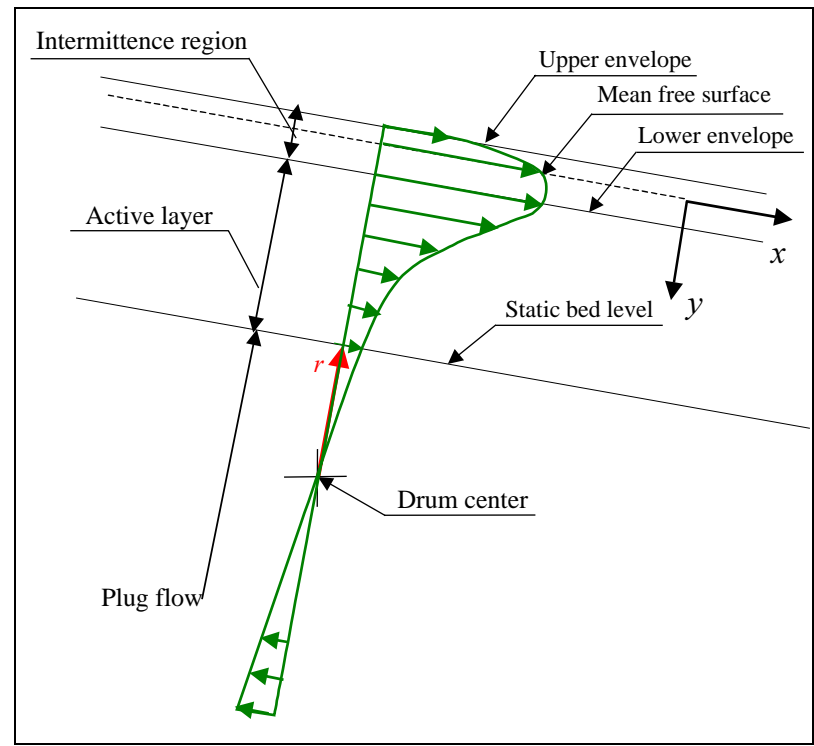

Fig. 3. Qualitative velocity profile and reference system in use. Upper and lower envelope of the intermittence region are determined by visual inspections; active layer, static bed level and mean free surface are defined on the basis of LDA data, as it appears in Fig. 4.

posed above, and flow slopes, obtained by visual analysis, are shown in Table 2 for different rotation speeds.

Figure 4 summarises as an example the most important results of the measurements performed with the LDA: velocity, "temperature" and data rate profiles along the vertical. On the same figure, the criteria used to distinguish the different flow regions (the intermittent presence layer, the dynamic and the quasi-static layer) are defined. The mean free surface elevation is determined as the elevation corresponding to $50 \%$ of the average LDA "data rate" when measured from the wall. The depth of the dynamic layer is assumed to be the depth corresponding to the drop in the grain "temperature" to $50 \%$ of the maximum, depth which represents the transition from a collisional to a frictional regime. Finally, the depth of the quasi-static layer is assumed to be the depth corresponding to the drop of the temporal mean velocity to $1 \%$ of the maximum.

The choice of the LDA data rate as criterion to determine the mean free surface elevation is related to the use of this parameter as an indicator of presence and concentration. In the case of particles greater than the measurement volume, it is not clear how the data rate reflects particle concentration and velocity. From the high data rate value obtained in the low velocity plug flow, it appears that velocity is not a relevant factor; concentration and mainly the presence of granular material seem to be the controlling factors. When a particle packed in the plug flow slightly moves from its position, produces a burst if intercepts with its roughness the measure volume. The highest value of the data rate at the static bed level is due to the high reflection produced by a percentage of mica in the granular mixture, which segregates in the radial direction and concentrates in correspondence with the centre 
Table 2. Results of measurements performed with $0.84-1.19 \mathrm{~mm}$ sand grains at different drum rotation speeds

\begin{tabular}{|c|c|c|c|c|c|c|c|c|}
\hline \multicolumn{9}{|c|}{ Measurements at $1.5 \mathrm{~mm}$ from the wall } \\
\hline $\begin{array}{c}\mathrm{n} \\
\mathrm{rpm}\end{array}$ & $\begin{array}{c}\theta \\
{\left[{ }^{\circ}\right]}\end{array}$ & $\begin{array}{c}q_{v} \\
{\left[\mathrm{~m}^{2} / \mathrm{s}\right]}\end{array}$ & $\begin{array}{c}q_{c} \\
{\left[\mathrm{~m}^{2} / \mathrm{s}\right]}\end{array}$ & $\begin{array}{c}v_{S} \\
{[\mathrm{~m} / \mathrm{s}]}\end{array}$ & $\begin{array}{c}h_{q s} \\
{[\mathrm{~mm}]}\end{array}$ & $\begin{array}{c}h_{d} \\
{[\mathrm{~mm}]}\end{array}$ & $\begin{array}{c}h \\
{[\mathrm{~mm}]}\end{array}$ & $F r$ \\
\hline 1 & 34.20 & $2.47 \cdot 10^{-4}$ & $1.14 \cdot 10^{-3}$ & 0.155 & 9.1 & 3.3 & 12.4 & 0.353 \\
\hline 1.5 & 34.20 & $4.81 \cdot 10^{-4}$ & $1.72 \cdot 10^{-3}$ & 0.207 & 10.0 & 4.1 & 14.1 & 0.435 \\
\hline 2 & 34.00 & $6.96 \cdot 10^{-4}$ & $2.29 \cdot 10^{-3}$ & 0.275 & 8.3 & 6.6 & 14.9 & 0.577 \\
\hline 2.5 & 33.50 & $9.86 \cdot 10^{-4}$ & $2.87 \cdot 10^{-3}$ & 0.319 & 8.3 & 7.5 & 15.8 & 0.669 \\
\hline 3 & 33.00 & $1.33 \cdot 10^{-3}$ & $3.45 \cdot 10^{-3}$ & 0.358 & 9.7 & 7.1 & 16.8 & 0.753 \\
\hline 3.5 & 33.00 & $1.62 \cdot 10^{-3}$ & $4.03 \cdot 10^{-3}$ & 0.425 & 7.0 & 10.6 & 17.6 & 0.892 \\
\hline 4 & 33.00 & $2.26 \cdot 10^{-3}$ & $4.61 \cdot 10^{-3}$ & 0.500 & 7.2 & 11.3 & 18.5 & 1.051 \\
\hline 4.5 & 32.50 & $2.66 \cdot 10^{-3}$ & $5.19 \cdot 10^{-3}$ & 0.606 & 9.6 & 10.6 & 20.2 & 1.262 \\
\hline 5 & 32.00 & $3.36 \cdot 10^{-3}$ & $5.71 \cdot 10^{-3}$ & 0.670 & 9.1 & 12.1 & 21.2 & 1.409 \\
\hline 5.5 & 31.00 & $3.94 \cdot 10^{-3}$ & $6.34 \cdot 10^{-3}$ & 0.723 & 8.6 & 13.7 & 22.3 & 1.518 \\
\hline 6 & 31.00 & $4.35 \cdot 10^{-3}$ & $6.92 \cdot 10^{-3}$ & 0.764 & 8.5 & 14.6 & 23.1 & 1.605 \\
\hline \multicolumn{9}{|c|}{ Measurements at $5.5 \mathrm{~mm}$ from the wall } \\
\hline 1 & & $5.80 \cdot 10^{-4}$ & & 0.203 & 8.7 & 7.0 & 15.7 & 0.517 \\
\hline 2 & & $1.10 \cdot 10^{-3}$ & & 0.305 & 9.1 & 9.1 & 18.2 & 0.721 \\
\hline 3 & & $1.83 \cdot 10^{-3}$ & & 0.416 & 10.0 & 10.9 & 20.9 & 0.918 \\
\hline 4 & & $3.43 \cdot 10^{-3}$ & & 0.584 & 9.2 & 13.4 & 22.6 & 1.241 \\
\hline 5 & & $4.84 \cdot 10^{-3}$ & & 0.707 & 9.3 & 15.3 & 24.6 & 1.440 \\
\hline 6 & & $6.26 \cdot 10^{-3}$ & & 0.845 & 9.3 & 17.2 & 26.5 & 1.657 \\
\hline
\end{tabular}

Legend:

$n=$ drum rotations per minute

$\theta=$ local flow slope with respect to the horizontal

$q_{v}=$ volumetric discharge per unit width obtained by integration of the velocity profiles assuming $C=C_{0}$

$q_{c}=$ volumetric discharge per unit width obtained by plug flow

$v_{s}=$ maximum flow velocity

$h_{q s}=$ thickness of the quasi-static layer (from the depth corresponding to $1 \%$ of the maximum flow velocity to the depth corresponding to $50 \%$ of the maximum granular "temperature")

$h_{d}=$ depth of the dynamic layer (from the depth corresponding to the $50 \%$ of the maximum granular "temperature" to the medium envelope)

$h=$ total flow depth $=h_{q s}+h_{d}$

$F r=$ Froude number evaluated as: $F r=v_{s}(g h)^{-1 / 2}$

of the drum. In the shearing region, where the concentration is near to the maximum and the presence is continuous, the data rate is approximately constant. Due to this observation, the data rate, assumed to be equal to $100 \%$ of presence, corresponds to the mean and not to the maximum in the active layer. In the intermittence region, the data rate decreases very rapidly as the presence of the material decreases. Thus, the definition of the mean free surface, as the depth corresponding to $50 \%$ of the average data rate, is the elevation at which grains are present for $50 \%$ of the measurement time.

\subsection{Side wall effects}

Even if the axial length of the drum is about one order of magnitude greater than the depth of the active layer, side wall effects have been recorded for every flow in a channel with walls. Friction, shearing and the side walls affect velocity values, mean free surface elevation, concentration in volume and grain size distribution across the channel. Some of these effects can be quantified comparing the discharge per unit width $q_{v}$ that can be obtained by integrating the velocity and concentration experimental profiles:

$q_{n}=\int C u d y$,

where $C$ is the volumetric concentration and $u$ is the horizontal velocity component at the flow height $y$, with the discharge per unit width $q_{c}$ derived from the plug flow:

$q_{c}=C_{0} \frac{\omega}{2}\left(R^{2}-r^{2}\right)$,

where $C_{0}$ is the volumetric concentration at rest obtained from measured porosity and equal to $0.58, R$ is the radius of the drum and $r$ is the radius corresponding to the bottom 


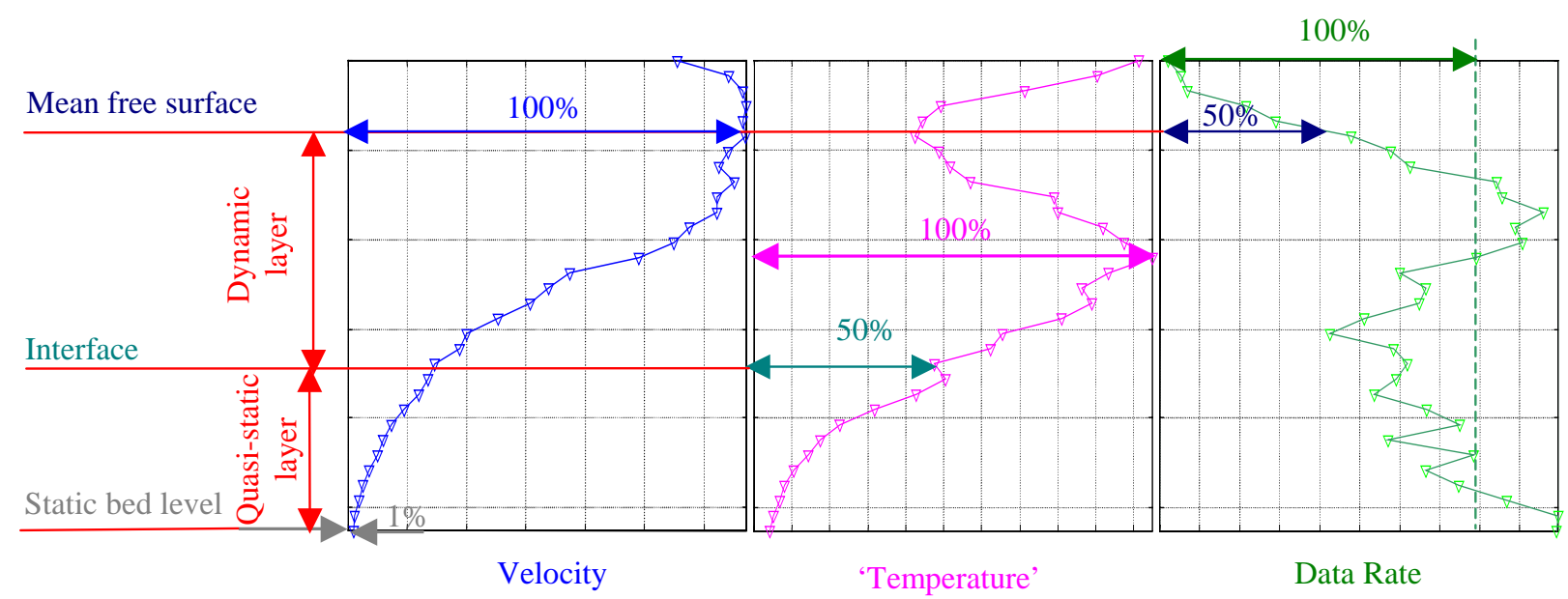

Fig. 4. Characteristic flow depths on the basis of the LDA data acquired entering from the wall. The active layer is divided into dynamic and quasi-static layer according to the "temperature" profile. The static bed level is defined where velocity drops to $1 \%$ of the free-surface value. The mean free surface is defined where the data rate drops to $50 \%$ of the typical value in the active layer.

of the active layer, represented in Fig. 3. Values obtained from Eq. (1) result much less than the values determined by Eq. (2), even assuming that $C$ is constant along the vertical and equal to $C_{0}$ (Table 2).

The concentration in volume is actually not constant along the vertical and is lower at the wall than across the channel due to intense shear at the glass wall. Image analysis, counting particles in the first layer near the glass wall per unit area, showed that concentration does not depend significantly on the rotation speed, but varies essentially along the vertical. The mean concentration varies at the wall from 0.58 at the flow bottom to 0.22 on the free surface, whereas on the free surface at the centre of the channel, it is about 0.43 .

Due to the friction at the walls, the velocity values obtained at a distance of $5.5 \mathrm{~mm}$ from the wall (Fig. 6) are higher than the values reached at $1.5 \mathrm{~mm}$ from the wall (Fig. 5) and the dynamic layer is deeper.

In comparing the mean dimensions of the particles at the walls and at the centre of the channel, a segregation mechanism appears, more marked at lower rotation speeds, with the finer material concentrated at the centre of the mid-channel section (Fig. 7).

In order to synthesise the wall effects on the threedimensional motion, Fig. 8 shows the distributions of velocity, mean flow height and volumetric concentration as a function of the distance from the wall. The values of the mean surface elevation $y_{m s}$ are derived in this case from:

$y_{m s}=\frac{\int y D_{R} d y}{\int D_{R} d y}$

where $D_{R}$ is the data rate at height $y$, obtained entering with the LDA obliquely from above; similarly, the mean freesurface velocities $v_{m s}$ are given by:

$v_{m s}=\frac{\int v D_{R} d y}{\int D_{R} d y}$ where $v$ is the velocity at the height $y$. In these measurements, the data rate goes to zero when the material is practically absent, such as on the top of the intermittence region, and when the measure point is too deep in the granular material, such as at the lower envelope of the intermittence region; a plot of the data rate along the vertical in this region gives an almost Gaussian curve. Since the penetration of the measuring system is limited, the mean data rate actually measures the percentage of time during which the free surface is a little above the point of measurement; therefore, the free-surface position is a little lower across the channel than the value obtained close to the wall.

In Fig. 8, free-surface velocity, mean free-surface elevation and volumetric concentration increase with increasing distance from the wall and reach the maximum value at the mid-channel width. The free-surface curvature suggests the presence of a mirror-like curvature at the flow bottom with flow depth increasing from the walls towards the centre of the channel.

\subsection{Flow regimes}

By increasing the drum rotation speed, quasi-stationary freesurface waves develop (Fig. 9), due to a change in the flow regime in the drum. The flow regime is stationary and uniform at low speeds, i.e. up to 3-3.5 rpm in our drum, when the flow becomes critical: the critical flow condition is evaluated by the authors, as in the case of a liquid, see Brennen et al. (1982) and Campbell et al. (1985). At higher speeds, stationary oscillations of the free surface develop due to oscillations of the interface between the dynamic and the quasistatic layer, with temporary stops in part of the underlying layers. At $6 \mathrm{rpm}$ the vertical displacement of the free surface is $12.5 \mathrm{~mm}$, of the same order of magnitude as the depth of the dynamic layer, and the instantaneous surface slope varies between $30^{\circ}$ to $39^{\circ}$, i.e. about $\pm 13 \%$. The formation of 


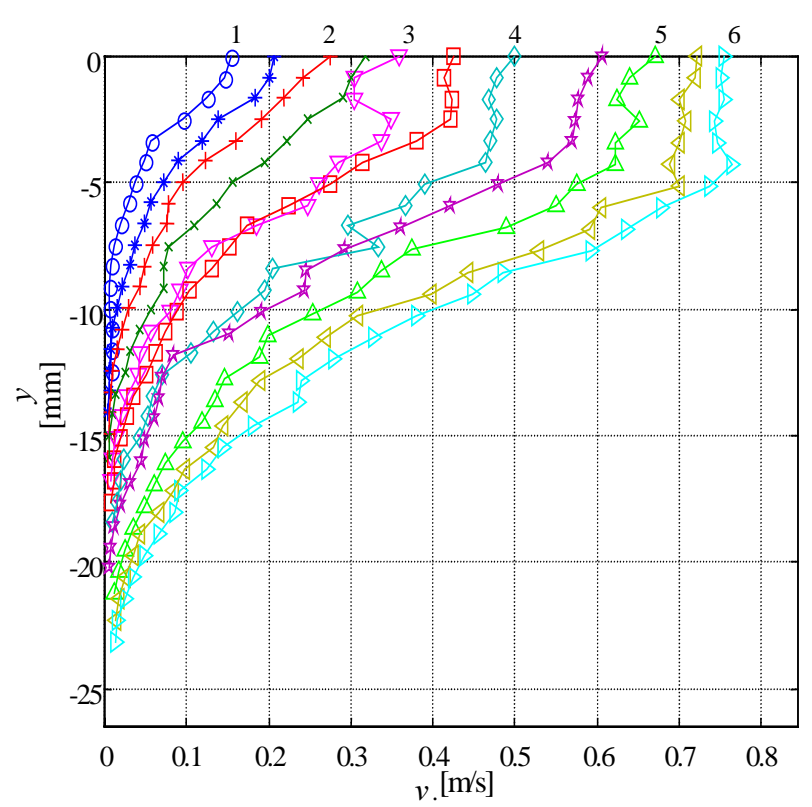

Fig. 5. Velocity $v$ profiles along the vertical $y$ measured at $1.5 \mathrm{~mm}$ from the wall, rotation speed: $1,1.5, \ldots, 5.5,6 \mathrm{rpm}$.

free-surface waves, in correspondence with Froude numbers greater than 1.0, agrees with the work of Lanzoni and Seminara (1993), even if it must be stressed that they reached this result in the case of a mixture of water and glass beads on a rigid and plane bottom, i.e. in conditions quite different from those of the present experiments.

\section{Experimental velocity profiles and theoretical ap- proximations}

This section compares the experimental velocity profiles and the derived parameters with existent theoretical approximations. Velocity profiles are first analysed comparing the experimental data with the velocity distributions given by Takahashi and a semi-empirical relation proposed by the authors (Sect. 4.1). This comparison highlights the presence of relevant free-surface oscillations, the effect of which is described in Sect. 4.2. Then, Sect. 4.3 considers the relation between surface velocity and discharge derived from plug flow, the only two data that are not affected by side wall effects. Experimental results are compared with the analytic results obtained by calibrating Takahashi's velocity approximation for stony debris flows and effects of free-surface waves on measurements are shown. Finally, discrepancies between the expected and measured values of the volumetric discharge are discussed in Sect. 4.4.

\subsection{Velocity distributions}

Takahashi (1991) suggested two different velocity profiles for stony debris flows over a rigid bottom:

$v(y)=v_{s}\left(1-\left(\frac{y}{h}\right)^{3 / 2}\right)$

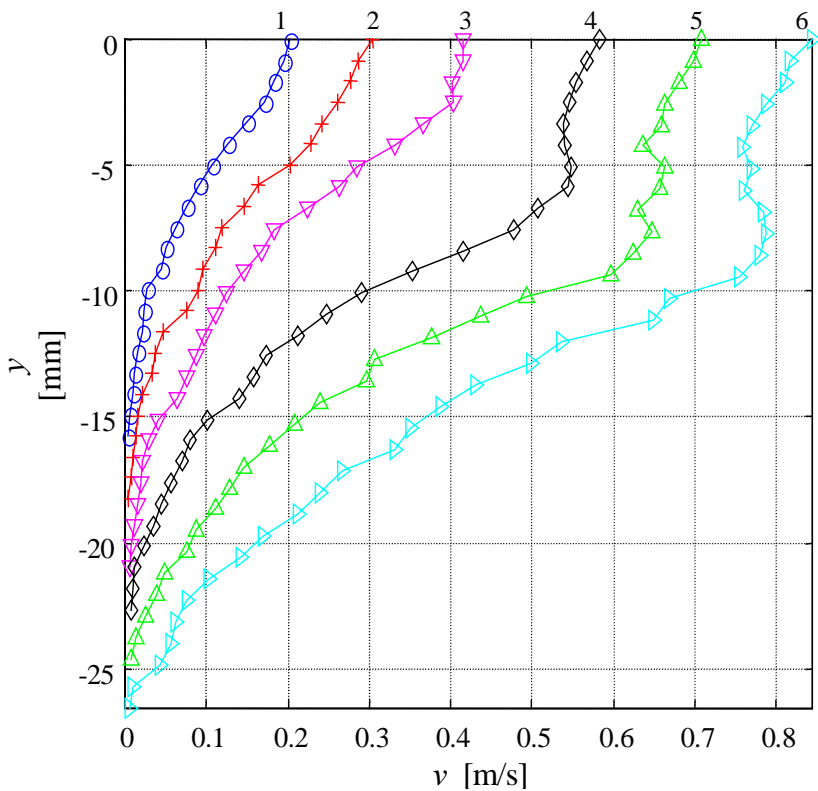

Fig. 6. Velocity $v$ profiles along the vertical $y$ measured at $5.5 \mathrm{~mm}$ from the wall, rotation speed: $1,2, \ldots, 5,6 \mathrm{rpm}$.

and over an erodible bottom:

$v(y)=v_{s}\left[1-\frac{1}{2}\left(\frac{y}{h}\right)^{3 / 2}\left(2+3\left(1-\frac{y}{h}\right)\right)\right]$.

The best adaptation of the experimental velocity profiles obtained by our measurements is given by the following semiempirical relationship:

$v(y)=v_{s}\left(1-\tanh \left(\frac{y}{h_{d}}\right)^{3 / 2}\right)\left(1-\left(\frac{y}{3 h_{d}}\right)^{5}\right)$.

Equations (5), (6) and (7) have several similar features: they depend only on two parameters, a depth scale factor $-h_{d}$ and $h$, respectively, and a characteristic velocity, the surface velocity $v_{s}$, and present the same kind of dependence on elevation near the free surface. Equations (5) and (6) are modified with respect to the original forms because the reference system used by Takahashi has the zero at the flow bottom, whereas our reference system has the zero in correspondence with the mean free surface (Fig. 3), since the flow bottom is the most uncertain parameter to be determined in our measurements. Moreover, the scale factor $h_{d}$ does not correspond, as Takahashi's scale factor $h$, to the total flow depth but represents the depth of the dynamic layer. Figures 10 and 11 show that the curve obtained from (7) is very close to Takahashi's curve for erodible bed (6) in the upper part, whereas in the lower part goes more slowly to zero. In Table 3, discharge and momentum coefficients are compared for all the approximations (5), (6), (7): the discharge coefficients obtained from Eqs. (5) and (6) are different because of the different scale factor, but the momentum coefficients are practically equal.

The experimental profiles go asymptotically to zero and near the bottom they present an inflection point, in analogy 


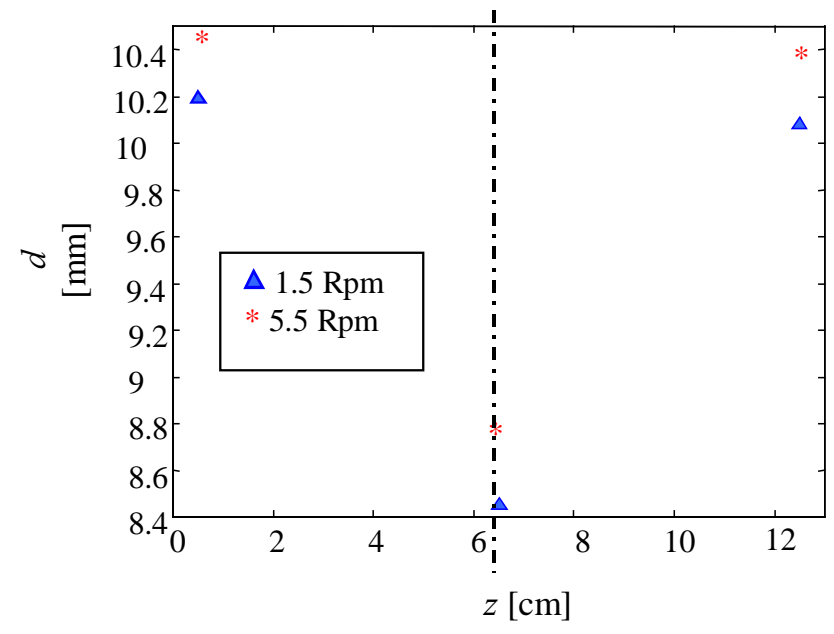

Fig. 7. Particle mean diameter $d$ versus channel width $z$ from the glass front wall.

with the results of Savage (1979), Ishida and Shirai (1979), Knight (1983), Takahashi (1991) and Egashira (2000). In the upper part of the dynamic layer, near the mean free surface, the velocity profiles present a flattening, at rotation speeds higher than $3.5 \mathrm{rpm}$, which can be justified with the presence of free-surface oscillations (see the following subsection). In fact, the instrument acquires data only in the presence of material, so the mean velocity values acquired in the intermittence region represent the mean velocity on the instantaneous surface and a little underneath: this value appears almost constant when the free-surface position varies.

The curves presented in Figs. 12 and 13 fit the experimental profiles using Eq. (7); the fitting curves agree with the experimental profiles until the lower envelope, then disagree in the intermittence region. In fact, these curves have been obtained by a weighted fitting of the data, with the assumption that the weights of the points at constant velocity in the region between the lower envelope and the mean free surface are negligible with respect to the weights of the points between the bottom and the lower envelope. This assumption is based on the interpretation that free-surface waves are present and propagate upstream, reducing mean measured velocity near to the free surface. In the following section, a rough representation of the phenomenon is given.

\subsection{Free-surface waves}

As pointed out in the previous subsection, the experimental velocity profiles disagree with Takahashi's profile in the lower part and in the flattening near the free surface. This fact, together with the difference between the discharge obtained by plug flow and by integration of velocity measurements (see Table 2), made us ask ourselves if measured velocities near the free surface are effectively correspondent to real values.

Form and extension of the experimental profile can be justified throughout the superposition of two different phenom-
Table 3. Discharge $C_{d}$ and momentum flow $\beta$ coefficients for different velocity distribution approximations. $C_{d}$ is the numerical coefficient given by the displacement thickness $h_{\Delta}$ scaled with the appropriate depth ( $h$ for Takahashi, $h_{d}$ for Lamberti)

\begin{tabular}{lcc}
\hline Approximation & $q=: h_{\Delta} v_{s}$ & $\beta:=\int v(y)^{2} d y /\left(q v_{s}\right)$ \\
\hline Takahashi, 1978 & $0.600 h v_{s}$ & 0.750 \\
Takahashi, 1991 & $0.428 h v_{s}$ & 0.687 \\
Lamberti, 2000 & $0.714 h_{d} v_{s}$ & 0.683 \\
\hline
\end{tabular}

ena on the basic flow field: surface flow displacements and surface waves.

Free-surface oscillations consist of vertical random displacements of the free surface, as it is evident in Fig. 9. At a first glance, free surface moves jointly with the interface between the dynamic and quasi-static layer, and the flow velocity profile is also assumed to translate with the free surface along the vertical. Flow is represented (Fig. 14a) as a fixed bottom until the lower envelope, on which oscillations of intensity $\eta$ occur; in this case, there is no longitudinal velocity gradient along the stream nor flow depth variation.

Free-surface waves propagate upstream almost as stationary waves, alternating between flow divergence and contraction over a fixed plane bed (Fig. 14b). For the sake of simplicity, the stream is supposed to have constant velocity along the vertical; in order to reproduce the stream discharge, the mean flow depth over the bed is equal to $h_{\Delta}$.

In order to check the reliability of these interpretations, we have reproduced both phenomena on Takahashi's profile (6). First, the profile was randomised, in order to represent freesurface oscillations. Equation (6) was rewritten as a function of the measure point elevation $y$ and vertical surface displacement $\eta$ from the mean free-surface position:

$v(y+\eta)=v_{s}\left(1-\frac{1}{2}\left(\frac{y+\eta}{h}\right)^{3 / 2}\left(5-3 \frac{y+\eta}{h}\right)\right)$

that is obviously valid in the range $\eta-y>0$. Vertical displacements $\eta$ are assumed to have a normal distribution with standard deviation $\sigma$, which is consistent with measurements performed across the channel. For each profile, the experimental values of $v_{s}, \eta$ and $\sigma$ were introduced in Eq. (8) and velocities at any fixed position were averaged, obtaining what we called a randomised profile. Near the free surface, velocity is averaged only over periods when the free surface is above the measure point in order to produce mean values $\overline{v(y)}$ that can be compared with mean measured velocity $\langle v(y)\rangle$. Indicating with $P(\eta)$ the probability of presence of the material at the elevation $\eta$, this average is given by:

$$
\overline{v(y)} \equiv \frac{\int_{y}^{\infty} v(y+\eta) d P(\eta)}{\int_{y}^{\infty} d P(\eta)} .
$$

Figure 15a shows one of the profiles obtained by a vertical randomisation of Takahashi's profile, together with the 


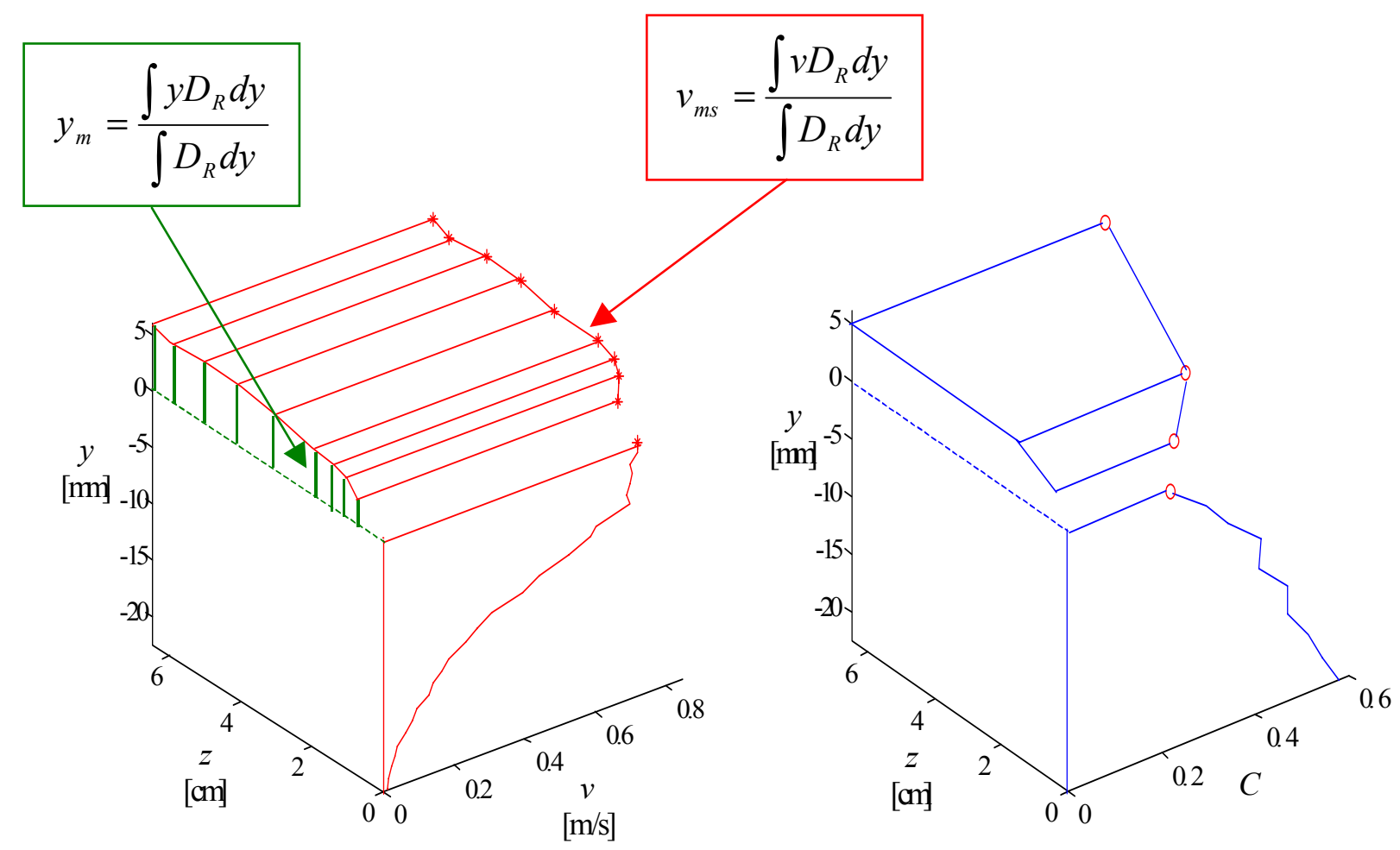

Fig. 8. On the left, velocity $v$ along the vertical $y$, measured at $1.5 \mathrm{~mm}$ from the wall, and on the free surface across the channel width $z$; the position of the mean free surface is also shown. The rotation speed of the drum is $5.5 \mathrm{rpm}$. On the right, concentration $C$ at the wall and on the free surface, observed at $2.5 \mathrm{rpm}$.

experimental velocity distribution, at the same rotating speed. The theoretical randomised profile presents the flattening near the free surface as the experimental curve; it decreases more slowly to zero near the bottom than the original theoretical profile, so it fits the experimental profile better in the lower part, as Eq. (7) proposed by the authors; however, it presents a rapid velocity decrease under the mean free surface that does not match the measurements.

In order to explain this disagreement, the effects of quasistationary surface waves on velocity profile are analysed. These waves propagate upstream, reducing flow velocities near the free surface: thus, mean measured velocities $\langle v(y)\rangle$ near the free surface do not represent the particle mean values, but reflect the condition that data are produced and averaged only when grains are present at the measure point $(\eta-y>0)$ and, therefore, mainly during the crest phase. Measured mean velocity values $\langle v(y)\rangle$ can be expressed as:

$$
\begin{array}{r}
\langle v(y)\rangle=\left\langle\frac{q}{h_{\Delta}+\eta}\right\rangle=\frac{q}{h_{\Delta}}\left\langle\frac{1}{1+\frac{\eta}{h_{\Delta}}}\right\rangle \\
=\frac{q}{h_{\Delta}}\left(\frac{\int_{y}^{\infty}\left(1+\frac{\eta}{h_{\Delta}}\right)^{-1} d P(\eta)}{\int_{y}^{\infty} d P(\eta)}\right)
\end{array}
$$

$$
\cong \frac{q}{h_{\Delta}}\left(\frac{\int_{y}^{\infty}\left(1-\frac{\eta}{h_{\Delta}}\right) d P(\eta)}{\int_{y}^{\infty} d P(\eta)}\right),
$$

where $q$ is the discharge per unit width, constant along the stream since waves are supposed to be stationary. Equation (10) gives, at each flow depth, the difference between the mean flow velocity $q / h_{\Delta}$ and the measured velocity; in particular, in correspondence with the mean free surface $(y=0)$, Eq. (10) becomes:

$\langle v(0)\rangle=v_{s}\left(1-\frac{\sigma}{h_{\Delta \sqrt{2 \pi}}}\right)$.

For each velocity profile, $\sigma$ was assumed to have the same value used in representing vertical surface displacements; $h_{\Delta}$ was evaluated following Table 3, where $h$ was imposed equal to the total observed flow depth; the value of $v_{s}$ was computed from Eq. (11). Then, these values were introduced in Eq. (8) and Takahashi's profile representing measured velocities $\langle v(y)\rangle$ was derived following Eq. (10). Figure 15b presents Takahashi's profiles obtained with this method, together with the experimental velocity distribution at the same rotating speed. Takahashi's distribution, randomised and modified in order to take into account the reduction effect of surface waves on measured velocities, shows a very close approximation to the experimental profile. 

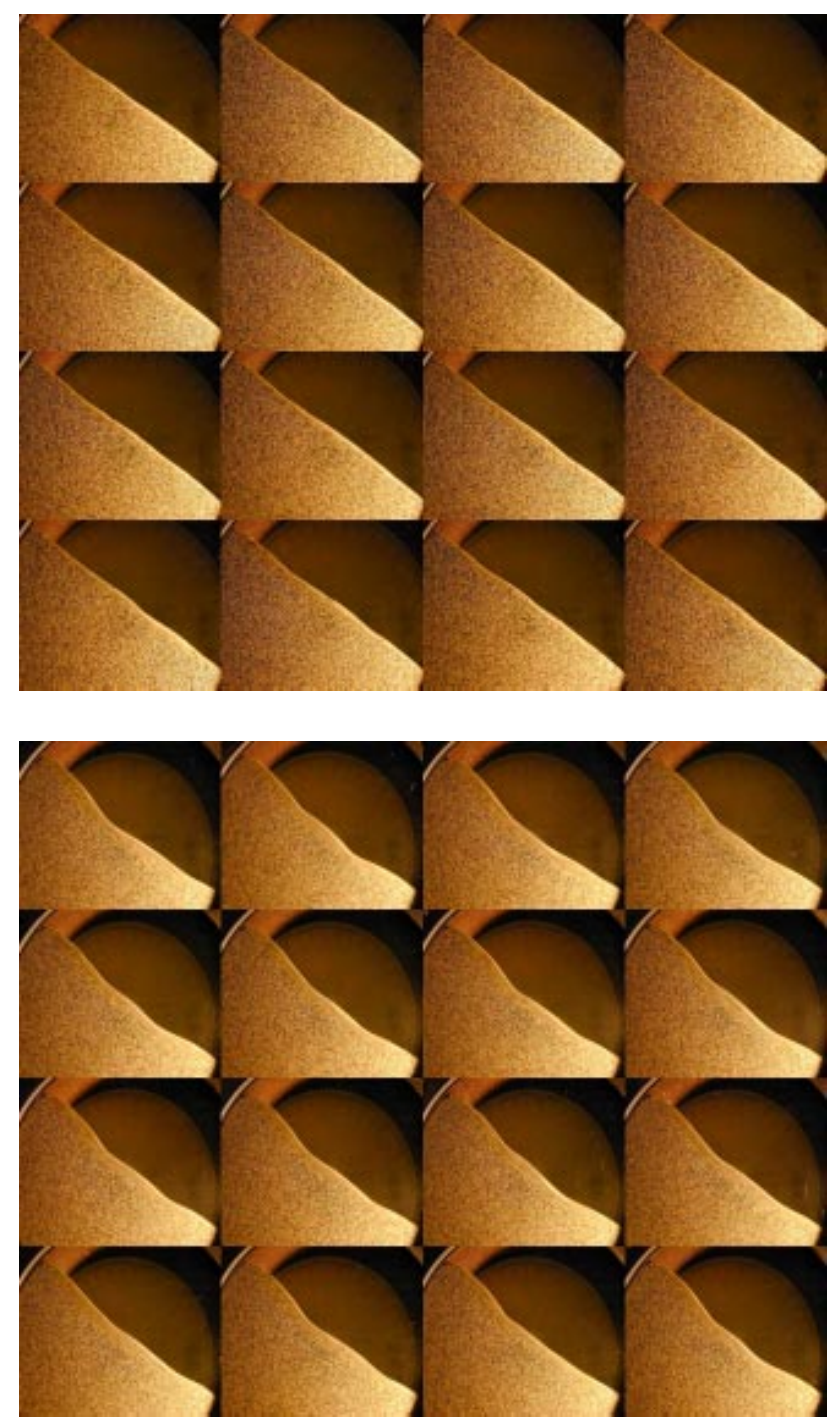

Fig. 9. Free surface and active layer profiles at $2 \mathrm{rpm}$ (upper) and at $6 \mathrm{rpm}$ (lower), at time intervals of $0.6 \mathrm{~s}$.

In conclusion, experimental results and Takahashi's profile show a good agreement considering that measurements are affected by free-surface oscillations, which modify the form of the profile near the free surface and near the static bed level. In order to quantify the contribution of the surface fluctuation of each type, it is necessary to determine the real correlation between surface elevation and velocity, remembering that no correlation means a prevail of random vertical displacements of the flow, whereas a negative correlation signifies a prevail of surface stationary waves. It was not possible to perform this kind of analysis with the available instrumentation because it requires synchronized measurements of velocity and free-surface elevation.

In the following subsection, the relevance of waves on the flow field is estimated through the comparison of the experimental relation between surface velocity and discharge that is obtained by neglecting and including the wave effect represented by Eq. (11).

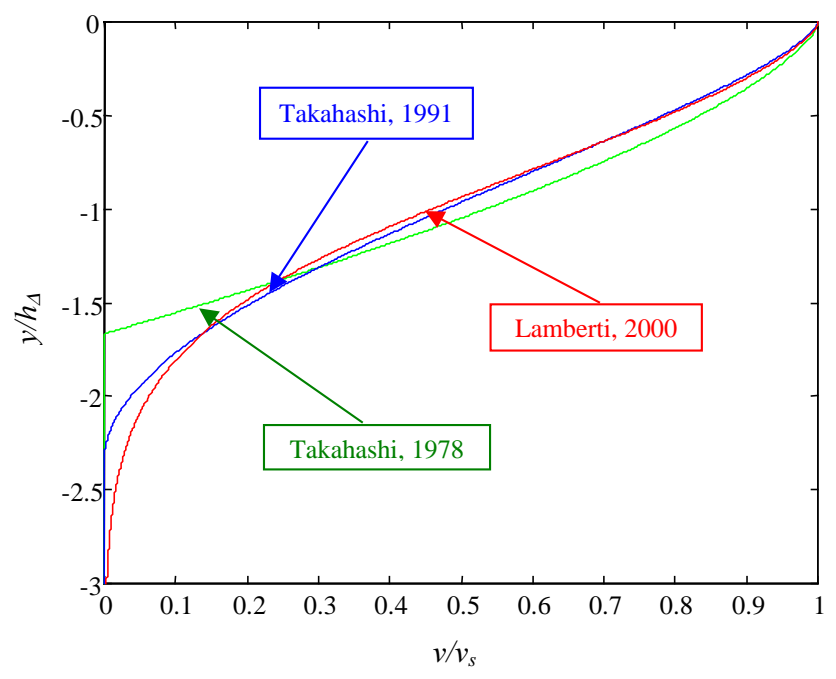

Fig. 10. Theoretical velocity distributions (origin at the freesurface): a comparison. Curves are presented with the elevation scaled by "displacement thickness" $h_{\Delta}$ and velocity scaled with free-surface velocity $v_{s}$.

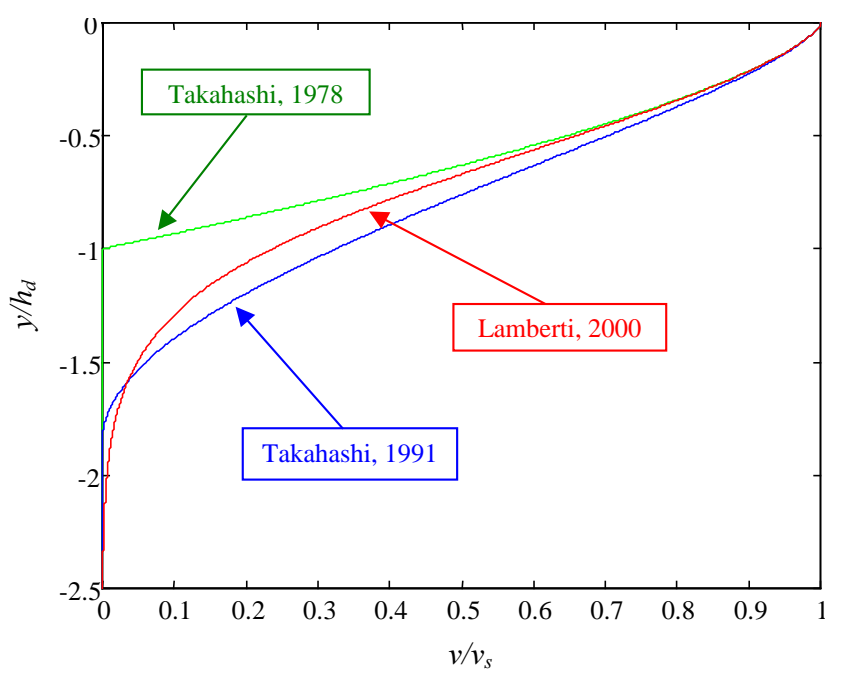

Fig. 11. Theoretical velocity distributions (origin at the freesurface): a comparison. Curves are presented with velocity scaled with the free-surface velocity $v_{s}$, and elevation scaled so that they have equal asymptotic behaviour near the free surface.

\subsection{Surface velocity variation with discharge}

Let us now consider only the experimental data that are not influenced by side wall effects: the mean free surface velocity at the centre of the channel, obtained by Eq. (4) and the discharge evaluated from Eq. (2).

The experimental results are compared with the theoretical relation that can be obtained by Takahashi's velocity distribution for stony debris flow, modified for the case of dry granular material:

$$
v(y)=\frac{2}{3 d}\left(\frac{g \sin \vartheta}{a \sin \alpha} C\right)^{1 / 2}
$$




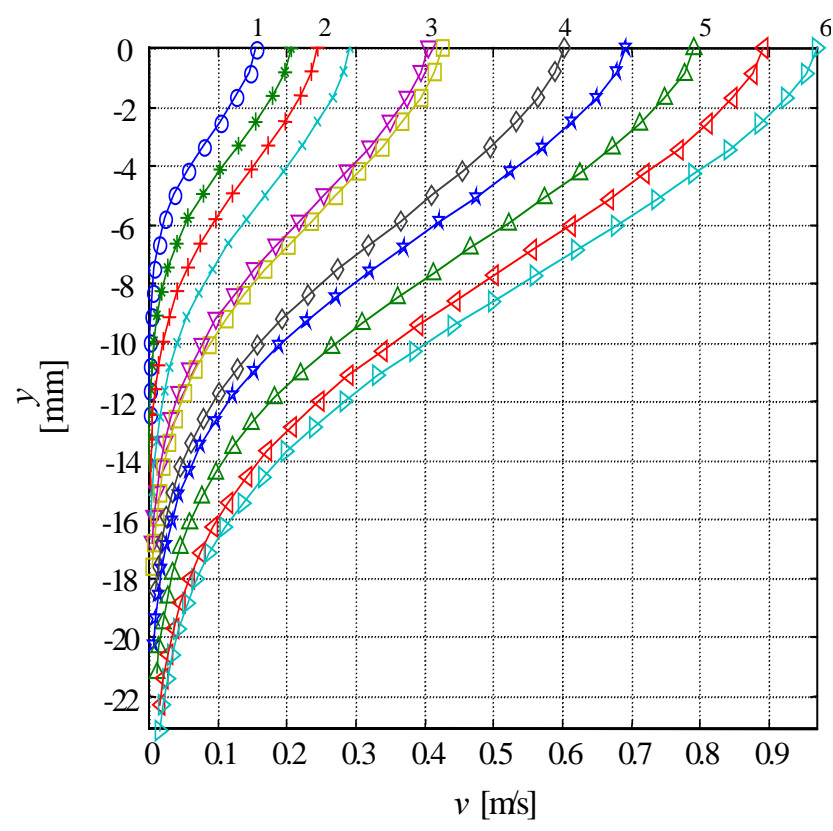

Fig. 12. Velocity profiles obtained following Eq. (7); curves fit experimental velocity measurments performed at $1.5 \mathrm{~mm}$ from the front wall, rotation speed: $1,1.5, \ldots, 5.5,6 \mathrm{rpm}$.

$$
\left[\left(\frac{C_{0}}{C}\right)^{1 / 3}-1\right] h^{3 / 2}\left[1-\left(\frac{y}{h}\right)^{3 / 2}\right],
$$

where $a$ is the Bagnold's constant, $\alpha$ is the dynamic friction angle, $\theta$ is the flow slope angle, $d$ is the particle mean diameter. In the following, we denote:

$$
\begin{aligned}
& K_{d}=\frac{2}{3 d}\left(\frac{g \sin \vartheta}{a \sin \alpha}\right)^{1 / 2} \\
& K_{c}=\sqrt{C}\left[\left(\frac{C_{0}}{C}\right)^{1 / 3}-1\right] \\
& v_{s}=K_{d} K_{c} h^{3 / 2} .
\end{aligned}
$$

For the sake of simplicity, relation (15) is derived following the Takahashi's approximation for rigid bed: in fact, the approximation for erodible bed has the same structure and the same dependence-law of surface velocity on flow depth but very complicated expressions for the constants $K_{d}$ and $K_{c}$ (see Takahashi, 1991).

Two different sets of parameters are used to calibrate Eq. (13) and Eq. (14), following Bagnold (1954):

$a=0.040$

$\tan \alpha=0.32$

and following Lamberti and Schippa (1999):

$a=0.035$

$\tan \alpha=0.78$.

From our experiments, the mean flow slope and the mean particle diameter are determined:

$\theta \approx 32^{\circ}$

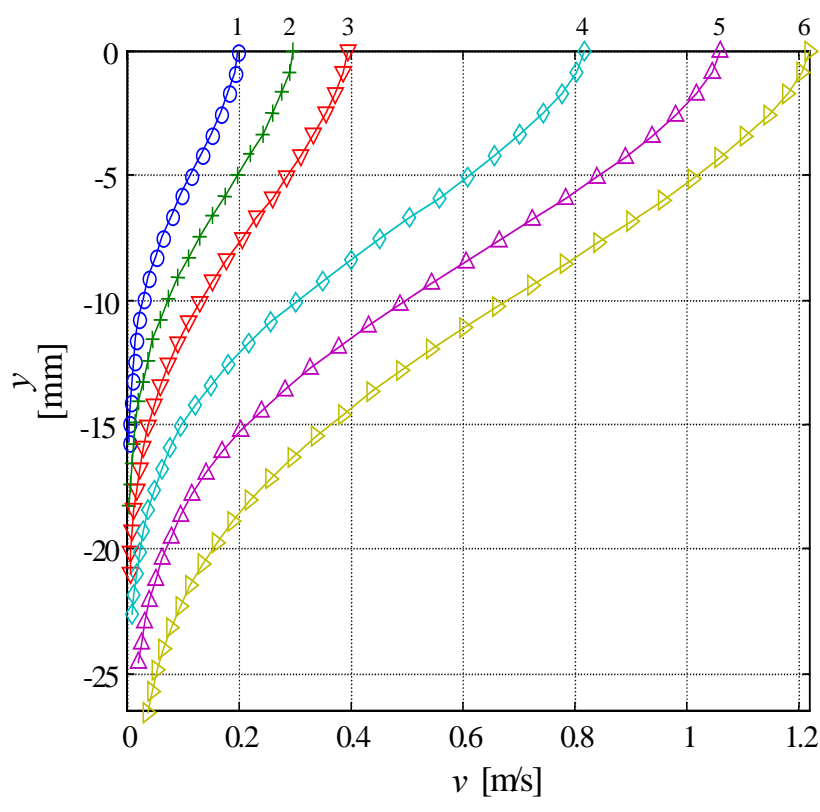

Fig. 13. Velocity profiles obtained following Eq. (7); curves fit experimental velocity measurments performed at $5.5 \mathrm{~mm}$ from the front wall, rotation speed: $1,2, \ldots, 5,6 \mathrm{rpm}$.

Table 4. Mean free surface concentration $C_{s}$ at the centre of the channel obtained by image analysis and concentration factor $K_{C}$ evaluated following Eq. (14)

\begin{tabular}{cccc}
\hline rpm & $C_{s}$ & $K_{c}$ & $C K_{c}^{-2 / 3}$ \\
\hline 1 & 0.441 & 0.031 & 5.167 \\
2 & 0.433 & 0.033 & 4.934 \\
3 & 0.407 & 0.039 & 4.302 \\
4 & 0.394 & 0.042 & 4.039 \\
5 & 0.379 & 0.045 & 3.771 \\
6 & 0.371 & 0.047 & 3.640 \\
\hline
\end{tabular}

$d=1 \mathrm{~mm}$.

The concentration in volume $C$ is evaluated as the arithmetic mean value between the concentration $C_{0}$ and the observed free-surface concentration $C_{s}$ (values of $C_{s}$ and $K_{c}$ in Table 4). The integration of the velocity distribution (12), assuming the concentration is nearly constant along the vertical, and the introduction of the discharge coefficient, defined in Table 3, give the following discharge-flow depth relation:

$q=\int C v d y \approx C \int v d y=0.6 K_{d} K_{c} C h^{5 / 2}$.

Substituting in Eq. (16) the expression for flow depth $h$ obtained by Eq. (15), the discharge free-surface velocity relation is determined:

$q=\frac{0.6 C v_{s}^{5 / 3}}{\left(K_{d} K_{c}\right)^{2 / 3}}$. 

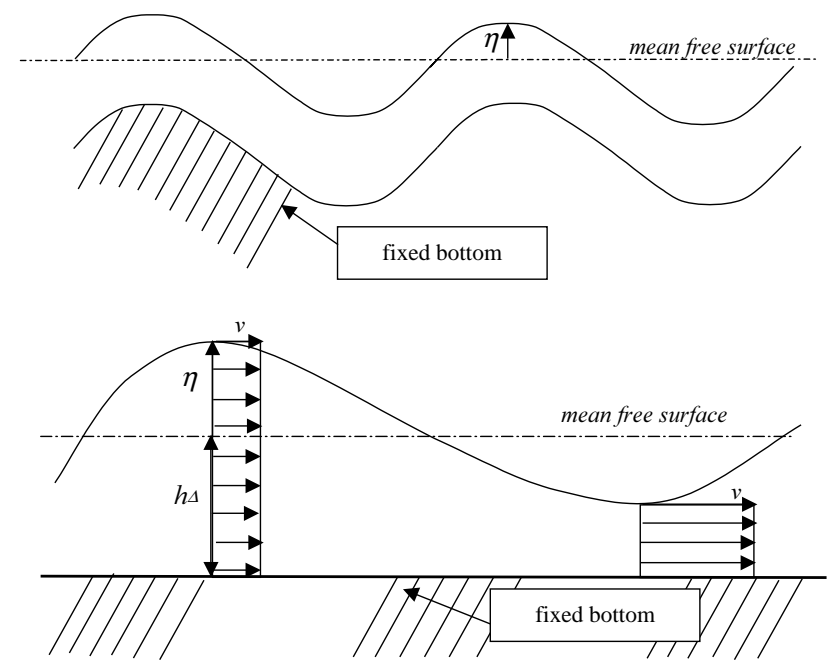

Fig. 14. Schemes of the two different mechanisms the superposition of which is used to justify form and extension of the experimental velocity profile (Sinuosity is exaggerated). (a) Surface vertical random displacements on a fixed buttom; (b) Surface waves on uniform flow depth $h_{\Delta}$ over a fixed bottom.

The theoretical relation (17) is presented in Fig. 16, imposing that $q$ is equal to $q_{c}$ and introducing both sets of parameters suggested by Bagnold (1954) and Lamberti and Schippa (1999). On the same figure, the experimental relation among $q_{c}$ and $v_{s}$ is shown, together with the curve representing $q_{c}$ versus the values of $v_{s}$ corrected following Eq. (11), in order to take into account the effects of free-surface waves. Both experimental curves show two typical slopes, one slightly lower than 0.6 for subcritical flows $(\mathrm{rpm}<2.5)$ and the other around 1.0 for critical and supercritical flows, when stationary waves are formed, whereas $v_{s}$ obtained by Eq. (17) results proportional to $q^{0.7}$. A reasonable fitting of the pure experimental data could be obtained by calibrating the parameters $\alpha$ and $a$ in a range of $a \arcsin \alpha$ that would not be consistent with direct estimates for the parameters (see, for instance, Lamberti and Schippa, 1999). The curve that relates obtained $v_{s}$ by Eq. (11) and $q_{c}$ fits well at the higher rotation speeds, when the flow becomes critical, showing the relevance of wave influence on measurements. Slight differences between experimental and theoretical values, in correspondence with supercritical flows, can be justified with the weakness of the simplified assumption that the concentration is constant along the vertical and equal to a mean value. In a real case, the problem of determining concentration and the related parameter $K_{c}$ remains open, since the slope-concentration relation suggested by Takahashi (1991) for debris flows also provides unrealistic estimates in the case of dry granular flows. In the case of subcritical flows, experimental and theoretical curves are significantly different: at these speeds, the ratio $h_{\Delta} / d$ is low (between 2.3 and 5.1 in our tests) if compared with the values assumed at critical and supercritical conditions (between 7.6 and 10.42) and the continuous scheme cannot represent the real flow conditions.
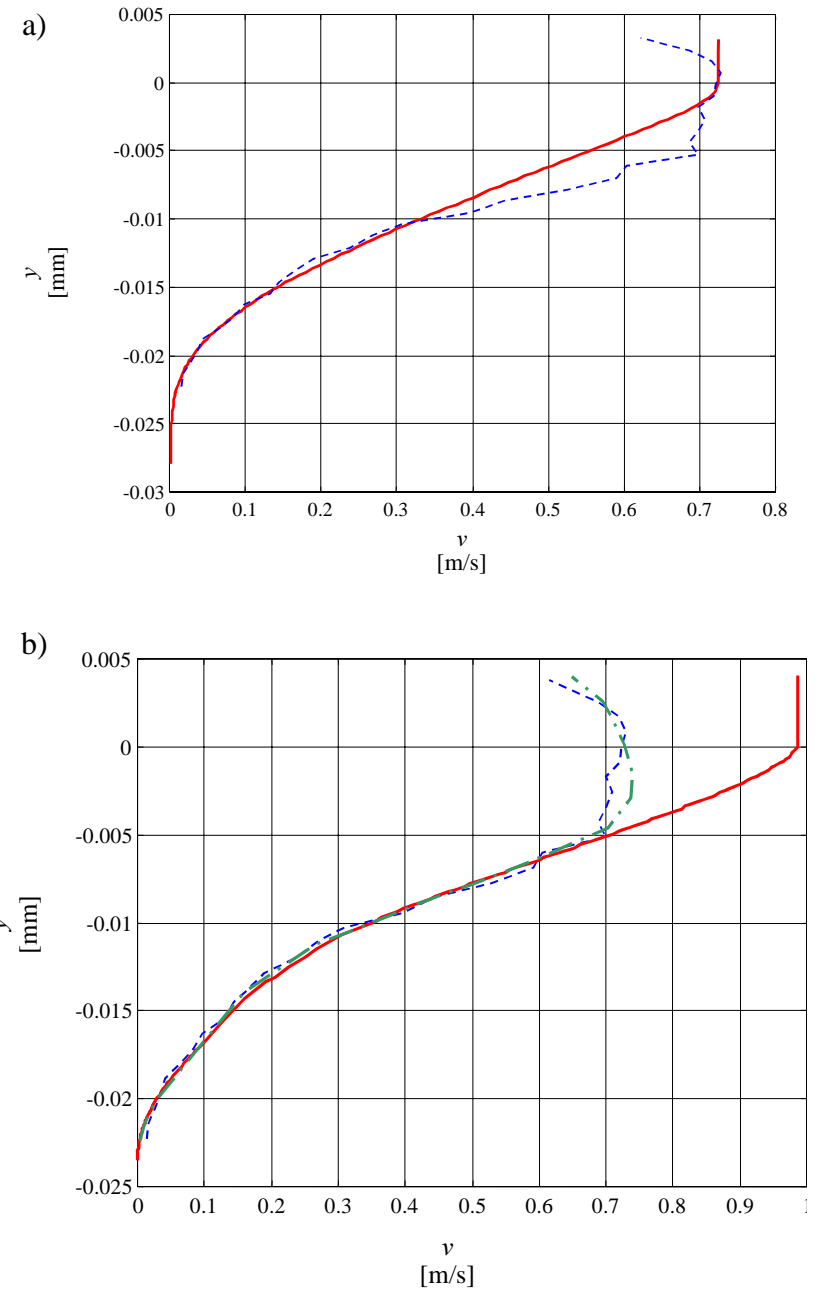

Fig. 15. Comparison between the experimental velocity profile in dotted line and Takahashi's randomised profile for erodible bed in continuous line, rotation speed $5.5 \mathrm{rpm}$; origin at the mean freesurface. (a) neglecting wave effect; (b) including wave effect. The dashed-dotted line in (b) corresponds to Takahashi's profile in continuous line modified following Eq. (10), in order to obtain measured velocities.

4.4 Discharge obtained by integrating velocity measurements and from plug flow

As can be deduced from Table 2, the discrepancy between the discharge obtained by integration of the measured velocity up to the mean free surface and the discharge obtained from plug flow is relevant. On the basis of Sects. 3.2, 3.3, 4.2 and 4.3 , this discrepancy is, in conclusion, due to:

- The 3D effects on depths and velocities. A factor 1.3 for velocities and 1.5 for depths is typical in our measurements; at low rotation speed the depth factor may reach a value around 2, explaining a factor of almost 3 in discharge.

- Surface waves. Unfortunately, the position of the free surface and the volumetric concentration could not be 


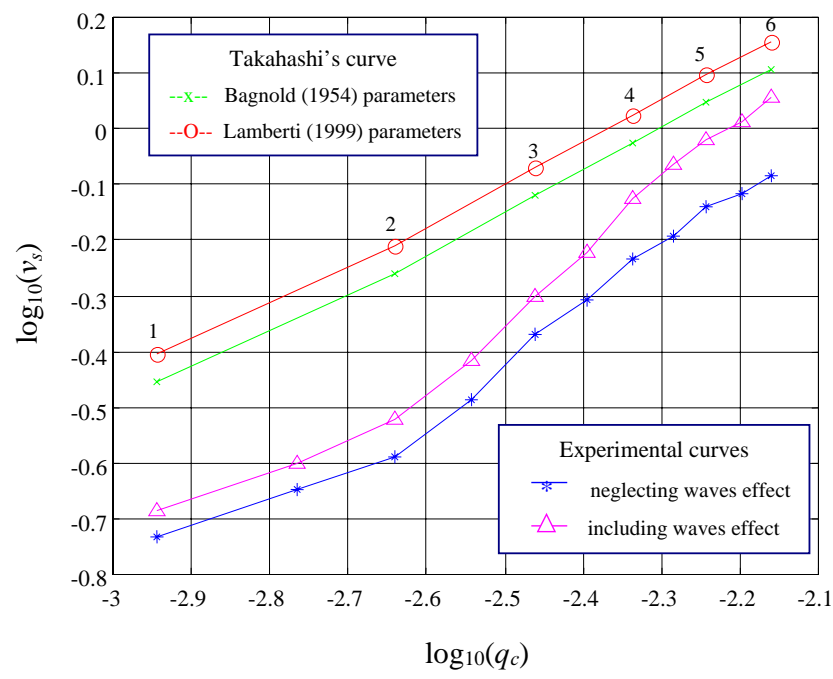

Fig. 16. Mean flow velocity on the free surface at the center of the channel $v_{s}$ versus the flow discharge obtained from the plug flow $q_{c}$, rotation speed range 1-6rpm. Comparison between the experimental data and the Takahashi's velocity-discharge relation. Takahashi's relation is calibrated using two sets of parameters: Bagnold (1954) and Lamberti (1999); the experimental curves are presented neglecting and including free-surface wave effect.

monitored with sufficient time resolution and the effects could not be exactly quantified.

- Effects of the choice of rheological parameters, which can be differently estimated according to Bagnold's (1954) experiments on neutrally buoyant wax spheres or to experiments performed by Lamberti and Schippa (1999) on sand grains.

- Simplifying assumptions, in particular, the assumption of a constant concentration along the vertical.

\section{Conclusions}

The analysis enlightened several discrepancies among theories and experiments that can be related to the following points:

- presence of 3D effects in the experimental setup;

- inadequacy of the measurements at the wall to represent bulk flow;

- weakness of the assumption that the concentration is constant along the vertical;

- existence and relevance of waves on transport relations.

In the drum, two different flow regimes can be distinguished: a uniform motion for subcritical flows and a motion with stationary surface waves for flows characterised by a Froude number near to 1 and greater.

The velocity profiles correlate well with a semi-empirical relation that essentially corresponds to the Takahashi's model for erodible bed: it contains only two parameters, a depth scale factor and a characteristic velocity, and presents the same kind of dependence on flow height near the free surface. However, the experimental profiles go asymptotically to zero and present a flattening near the mean free surface, in case of critical and supercritical flows, due to free-surface waves.

Form and extension of the experimental profiles can be justified throughout the superposition of two different phenomena on the basic flow field: vertical random displacements of the flow and almost stationary surface waves. Takahashi's distribution for erodible bed, randomised along the vertical and modified in order to take into account the reduction effect of surface waves on measured velocities, shows a very close approximation to the experimental velocity profiles.

In order to avoid side wall effects, the relation between surface velocity at the centre of the stream and discharge is analysed and compared with the relation proposed by Takahashi. The Takahashi's power-law relation has exponent 0.7, whereas the experimental relation shows two different slopes in correspondence with the change in the flow regime: approximately 0.6 for subcritical flows and around 1.0 for critical and supercritical flows. The correction of experimental velocity values, taking into account the presence of surface waves, allows for a good fitting of theoretical and experimental data for supercritical flows; in the case of subcritical flows, flow depth is small compared to grain size and the continuous scheme does not presumably represent the real flow conditions.

\section{References}

Bagnold, R. A.: Experiments on a gravity-free dispersion of large solid spheres in a Newtonian fluid under shear, Proceedings Royal Society London, A, 225, 49-63, 1954.

Boateng, A. A. and Barr, P. V.: Granular flow behaviour in the transverse plane of a partially filled rotating cylinder, J. Fluid Mechanics, 330, 233-251, 1996.

Brennen, C. E., Sieck, K., and Paslaski, J.: Hydraulic jumps in granular material flow, Powder Technology, 35, 31-37, 1982.

Campbell, C. S., Brennen, C., and Sabersky, R. H.: Flow regimes in inclined open channel flows of granular materials, Powder Technology, 41, 77-82, 1985.

Cantelaube, F. and Bideau, D.: Radial segregation in a 2-D drum: an experimental analysis, Eurphys. Lett., 30, 133-138, 1995.

Durst, F. and Ruck, B.: Effective particle size range in Laser Doppler Anemometry, Experiments in Fluids, 5, 5, 305-314, 1987.

Egashira, S., Itoh, T., and Takeuchi, H.: Transition mechanism of debris flow over rigid bed to erodible bed, J. Phys. and Chem. Earth, submitted, 2000.

Franklin, F. C. and Johanson, L. N.: Flow of granular flow material through a circular orifice, Chem. Eng. Sci., 4, 119-129, 1955.

Knight, P. C.: The role of particle collisions in determining high strain rate flow behaviour, Proc. Int. Symp. On the Role of Particle Interactions in Powder Mechanics, Eindhoven, 172-182, 1983. 
Kononenko, V. L.: Precision sizing of moving large particles using diffraction splitting of Doppler lines, Proceedings of SPIEThe International Society for optical engineering, 3736, 357364, 1999.

Ishida, M. and Shirai, S.: Velocity distributions in the flow of solid particles in an inclined open channel, J. Chem. Eng. Jpn., 12, 46-50, 1979.

Lanzoni, S. and Seminara, G.: Debris Waves, Proc. of XV IAHR Congress, Tech. Sess. B, 79-85, 1993.

Lanzoni, S. and Tubino, M.: Rheometric experiments on mature debris flows, Proc. of XV IAHR Congress, Tech. Sess. B, 47-54, 1993.

Naqwi, A. and Durst, F.: Light scattering applied to LDA and PDA measurements. Part 1 , theory and numerical treatments, Particle and Particle Systems Characterization, 8, 4, 245-258, 1991.

Naqwi, A. and Durst, F.: Light scattering applied to LDA and PDA measurements. Part 2, computational results and their discussion, Particle and Particle Systems Characterization, 9, 1, 66-80, 1992.
Rasmussen, K. R. and Iversen, J. D.: Grain Dynamics and WindFlow in a Variable Slope Wind Tunnel, in: Sustainable development in Arid Zones, Vol. 1, (Eds) Omar, S. A. S., Misak, R., and Al-Ajmi Balkema, D., Rotterdam, 1996.

Savage, S. B.: Gravity flow of cohensionless granular materials in chutes and channels, J. Fluid Mech., 22, 385-400, 1979.

Schippa, L. and Lamberti, A.: Experiments on the rheology of a dense granular flow, Debris Flow Risk Project Final Report, 2, 11.1-11.34, 1999.

Takahashi, T.: Debris Flow, IAHR monograph series, Balkema, 1$165,1991$.

Taniere, A., Boulet, P., and Oesterle, B.: Analysis of Laser Doppler Anemometry measurements: saltation effects on the behaviour of solid particles, Proceddings of the 7th International Conference of Laser Anemometry: Advances and Applications, Karlsruhe, 737-744, 1997.

Zanuttigh, B.: Deflusso di materiali granulari: analisi sperimentale e modellazione numerica, Degree Thesis, in italian, 1998. 